\title{
Image segmentation using dense and sparse hierarchies of superpixels
}

\author{
Felipe Lemes Galvão ${ }^{\mathrm{a}}$, Silvio Jamil Ferzoli Guimarães ${ }^{\mathrm{b}}$, Alexandre Xavier Falcão ${ }^{\mathrm{a}}$ \\ ${ }^{a}$ University of Campinas , Institute of Computing, Laboratory of Image Data Science, Av. Albert Einstein, 1251, \\ Campinas-SP, 13083-852, Brazil \\ ${ }^{b}$ Pontifical Catholic University of Minas Gerais, Computer Science Department, Belo Horizonte-MG, 31980-110, \\ Brazil
}

\begin{abstract}
We investigate the intersection between hierarchical and superpixel image segmentation. Two strategies are considered: (i) the classical region merging, that creates a dense hierarchy with a higher number of levels, and (ii) the recursive execution of some superpixel algorithm, which generates a sparse hierarchy with fewer levels. We show that, while dense methods can capture more intermediate or higher-level object information, sparse methods are considerably faster and usually with higher boundary adherence at finer levels. We first formalize the two strategies and present a sparse method, which is faster than its superpixel algorithm and with similar boundary adherence. We then propose a new dense method to be used as post-processing from the intermediate level, as obtained by our sparse method, upwards. This combination results in a unique strategy and the most effective hierarchical segmentation method among the compared state-of-the-art approaches, with efficiency comparable to the fastest superpixel algorithms.
\end{abstract}

Keywords: Superpixel Segmentation, Hierarchical Image Segmentation, Image Foresting Transform, Iterative Spanning Forest, Graph-based Image Segmentation, Irregular Image Pyramid 2010 MSC: 00-01, 99-00

\section{Introduction}

Superpixel segmentation has become an important low-level task in various image processing and computer vision applications [1, 2, 3. Superpixels are simply connected image regions with perceptually similar characteristics, often used to reduce the computational cost of higher-level image

Email addresses: felipe.galvao@students.ic.unicamp.br (Felipe Lemes Galvão), sjamil@pucminas.br (Silvio Jamil Ferzoli Guimarães), afalcao@ic.unicamp.br (Alexandre Xavier Falcão) 
operations given the lower number of image primitives [4]. On the other hand, a hierarchical image segmentation (or hierarchical segmentation) is a nested collection of progressively coarser image partitions. It enables multi-scale image analysis with applications to hierarchical scene annotation [5], co-saliency detection [6], and co-segmentation [7, 8]. Our focus is on hierarchical segmentation methods in which each individual scale can be used as an effective superpixel segmentation.

Classical algorithms for hierarchical image segmentation build a hierarchy through a series of operations such as region merging, which joins two or more connected regions into a single one, or region splitting, which separates a region into two or more smaller connected regions [4, 9, 10. By definition, each step in those algorithms can be seen as a new segmentation scale of a high number of observation scales - hierarchical levels that vary from a higher number of regions (the finest scale) to a lower number of regions (the coarsest scale) 1 Thus, we refer to those as dense hierarchies and dense hierarchical image segmentation methods (or simply dense methods).

Despite the existence of many dense methods with good image segmentation results, most of the related literature is focused on exploiting intermediate or higher-level object information. As a result, in the context of superpixel segmentation, we show that dense methods often present prohibitive execution time and poor boundary adherence at finer scales. Notable exceptions with competitive superpixel segmentation results are [11] and [12].

Superpixel algorithms were originally designed to create an image partition from its pixel set [13, 14, 15. Some of these algorithms can be easily executed recursively on each current image partition to create the next coarser image partition for hierarchical image segmentation [16, 17, 18. This allows the addition of a new coarser partition to the hierarchy at each iteration (see Figure 1), generally with significantly less number of regions than the previous one and overall creating a relatively small number of observation scales ${ }^{2}$ For that reason, we call those sparse hierarchies and sparse hierarchical image segmentation methods (or simply sparse methods).

Being derived from the superpixel literature, sparse methods fit the criteria for a hierarchy of superpixel partitions more naturally. Their main drawback is the limited granularity of the

\footnotetext{
${ }^{1}$ Any number of regions between two and the number of regions in the finest scale, assuming that exactly two regions are merged at a time (or a region is split into exactly two regions), which is the case for most region merging or region splitting algorithms.

${ }^{2}$ Note that this can be considered a particular type of irregular image pyramid [19, but here we avoid this denomination to focus on the superpixel segmentation context.
} 


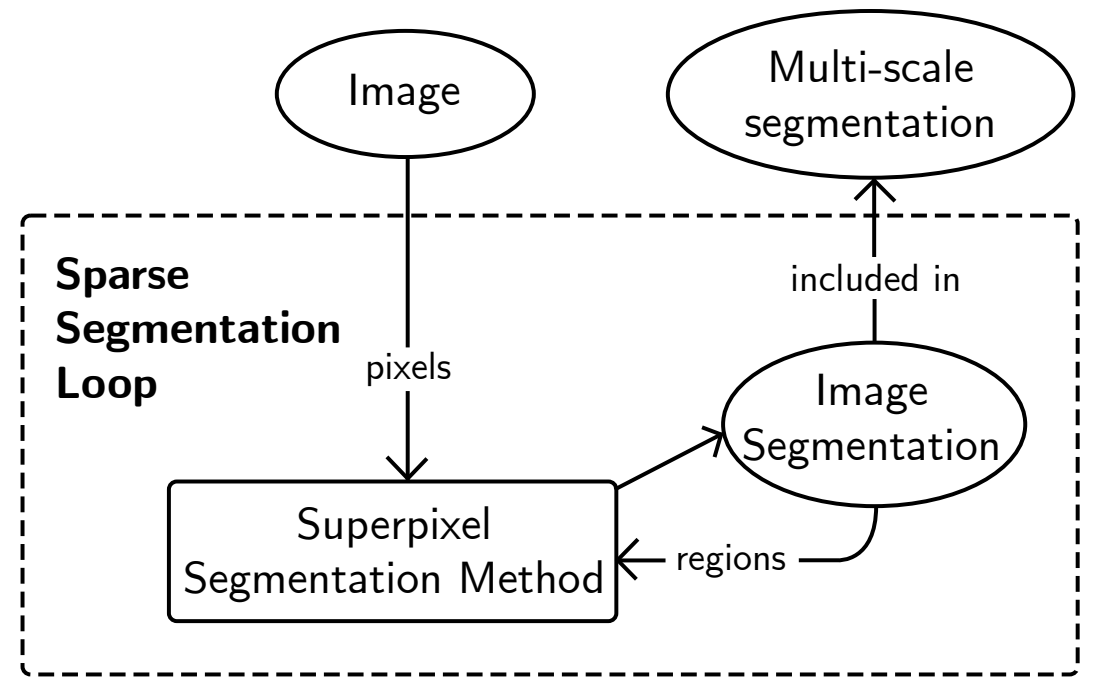

Figure 1: Overview of the typical pipeline of a superpixel-based sparse hierarchical image segmentation method. Image pixels or regions from a current image partition serve as input to some superpixel algorithm. At each iteration, the recursive loop adds a new segmentation scale coarser than the previous one to the hierarchy under construction.

hierarchical representation, especially when considering coarser scales where superpixels are similar in size to image objects.

In Figure 2, we highlight the difference between a single segmentation step of a dense and a sparse method, respectively. Note that the two approaches are not mutually exclusive; various dense methods start off from some initial set of superpixels [9, 20, 21].

The primary goal of this work is to formalize and review both categories of hierarchical image segmentation methods, dense and sparse, with an emphasis on those methods that obtain effective superpixel partitions. Our contributions in each category are described next.

In the context of sparse methods, we are particularly concerned with presenting how an existing superpixel algorithm can be modified to create a sparse hierarchical segmentation. The benefits are: (i) the possible improvement of the superpixel segmentation results, which can be obtained efficiently and using richer region-based features, and (ii) the extension of the superpixel algorithm to multiscale image analysis. For that, we propose a hierarchical version of the Iterative Spanning Forest (ISF) superpixel segmentation framework [15. This part is an extension of our previous work 18. 


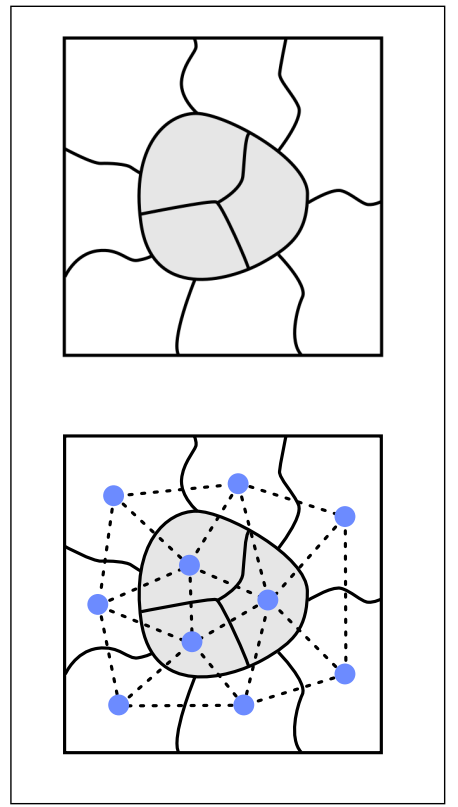

(a)

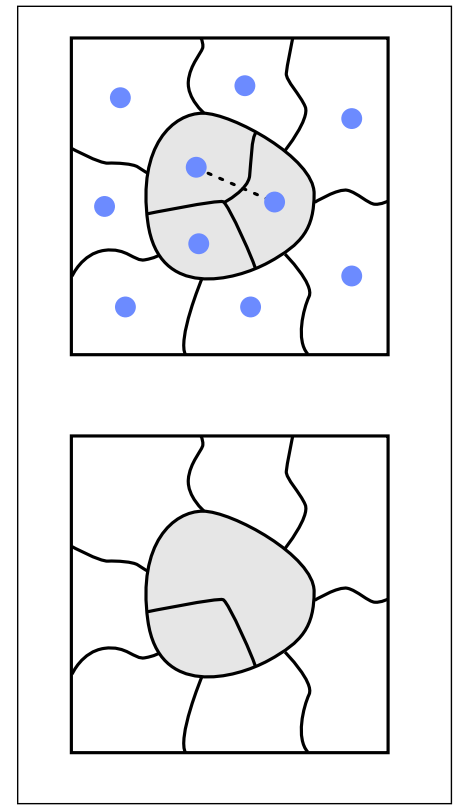

(b)

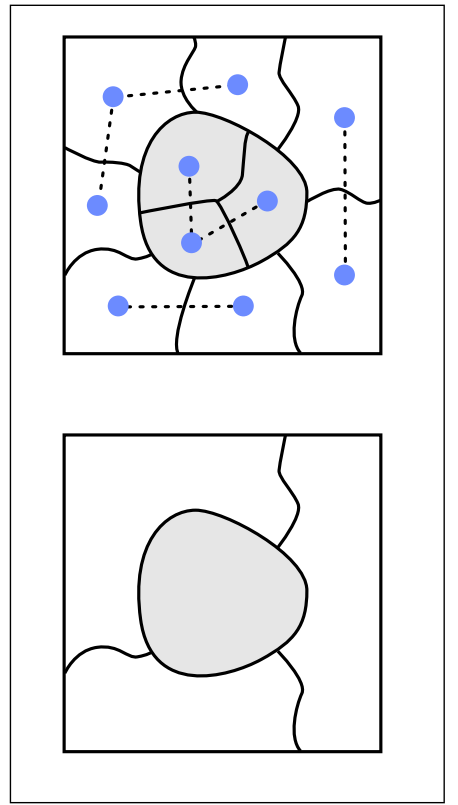

(c)

Figure 2: Comparison between a single segmentation step of dense and sparse methods using bottom-up graph-based examples. The difference is analogous to other grouping and partitioning algorithms. (a) An initial segmentation (top) and the corresponding region adjacency graph (RAG at the bottom) for both the dense and sparse approaches. (b) A single iteration of a dense method based on region merging: a pair of adjacent regions is selected for merging (top), and the number of regions decreases by one in the resulting segmentation (bottom). (c) A single iteration of a sparse method: an $n$-way partition of all nodes is computed (top), and the number of regions decreases down to $n$ in the resulting segmentation (bottom).

Here we show that our previous experimental settings were insufficient for a practical hierarchy with more than two observation scales ${ }^{3}$, and present a more suitable alternative. Additionally, our previous work only compared the hierarchical version against the original ISF methods; here we evaluate it against other state-of-the-art superpixel methods.

Beyond making ISF a hierarchical framework, we show that our proposed algorithm generates the entire sparse hierarchy faster than a single ISF segmentation and, as compared to existing sparse hierarchical segmentation methods, it benefits from allowing a fine control over each generated

\footnotetext{
${ }^{3}$ In [18], we were not focused on the hierarchical representation in itself and only used a simple hierarchy with two observation scales to exploit some properties of ISF.
} 
observation scale while not requiring any post-processing to ensure connected superpixels.

As previously mentioned, sparse hierarchies are not ideal when dealing with coarser scales. With that in mind, we also propose a new region-merging algorithm intended to build a dense hierarchy criterion, we show that, when it is used as post-processing to our proposed sparse method, the new region-merging algorithm beats the state-of-the-art superpixel segmentation methods in boundary adherence. Furthermore, our region-merging algorithm is fast, unsupervised, easy to implement, and can be used with any existing superpixel segmentation method.

In summary, the two main contributions of this work are: (i) proposing a hierarchical segmentation method in which the number and regularity of regions in each level can be easily defined; and (ii) proposing a strategy for merging regions according to a specified criterion, which can be used to create dense hierarchies from the intermediate level to above.

This paper is structured as follows. In Section 2, we present a review of hierarchical image segmentation and other related works. In Section 3, we describe important concepts related to our proposal. In Section 4, we introduce the proposed sparse and dense hierarchical segmentation methods, respectively. In Section 5 , we evaluate our proposed methods against the state-of-the-art hierarchical and superpixel segmentation methods. Finally, in Section 6, we state our conclusions and discuss future work.

\section{Related work}

In this section, we review the literature on hierarchical image segmentation, including both dense and sparse methods. We only cover superpixel algorithms associated with those categories. A broader review of superpixel segmentation can be found in [22].

\subsection{Dense hierarchical segmentation}

Being as old as the image segmentation problem itself, dense methods have a fairly extensive literature that is out of the scope of this work. Thus we focus on recent methods related to the superpixel literature that either use region splitting or region merging.

In [10, the spectral graph framework is used to create a region splitting method where each split corresponds to a bipartition of the underlying pixel graph with their proposed normalized cut. 
small partitions. An exact solution is NP-hard, and their approximation still relies on solving a complex eigenproblem, making it fairly slow in practice. It is notably important for its use in the work that coined the term superpixel [23], and for being part of the edge detector used in the method we describe next.

In [9], an ultrametric contour map (which is an alternative representation for a hierarchy of segmentations) is created by first generating an initial over-segmentation with a combination of the $\mathrm{gPb}$ edge detector and oriented watersheds (gPb-owt), followed by greedy merge operations weighted by the underlying border evidence across regions. The main advantage of the method is combining global information from top-down approaches and local information from bottom-up used, and the authors propose a method to speed up its computation. Additionally, the results are improved by updated contour cues and the use of image pyramids.

In [4, the gPb-owt is also used as a starting point, but the region merging step is based on training a cascade classifier which considers different scales of analysis independently. Based on the superior performance of the cascade classifier when compared to a single one for all scales, they show the importance of adapting the image segmentation objective function according to the scale of analysis. They also introduce the distinction for what they call the "superpixel regime" and the "large segment regime" to bridge the evaluation of methods generating intentional oversegmentations and methods trying to capture high-level information, respectively. It is worth to mention that this distinction was also used in our work and is better detailed in the experimental analysis (see Section 5).

For the specific context of methods that were designed with superpixel segmentation in mind, notable methods include the entropy rate superpixel segmentation (ERS) [1] and the Superpixel Hierarchy (SH) [12].

ERS introduces a greedy criterion based on the entropy of random walks, including a balancing term to encourage similarly sized superpixels. The method handles the processing time problem by employing sub-modular functions, allowing an efficient update of their heap structure. However, the restriction on using sub-modular functions also limit the customization of the method.

SH builds the image segmentation by computing a minimum spanning tree (MST) with Borůvka's MST-based methods, the method is very fast. It also incorporates hierarchical information by 
aggregating region features into a single new node (and corresponding new edges) each time the number of regions is reduced in half (one iteration of the algorithm). It starts with the mean color as a feature in finer scales and color histograms in coarser ones. Its main drawback is the lack of explicit control over the segmentation regularity.

For the remaining methods we cover, some superpixel segmentation is used as the starting point, benefiting from richer region features and reduced search space to employ more costly operations.

In [20], starting from the result of an ERS segmentation, they extract for each region the number of pixels, the mean pixel color, and the mean of the squared color. Then, using a fuzzy bi-partition operation that maximizes entropy and enforces connectivity, their method creates a hierarchical segmentation scheme over the superpixel topology. The process is guided by selecting at each step the region whose bi-partition minimizes the mean squared error comparing the original image to the mean pixel color from the segmented regions.

In [21, an initial oversegmentation of the image is first computed with the watershed algorithm [25] over the output of the $\mathrm{gPb}$ edge detector ${ }^{4}$ and then a greedy merging algorithm is performed over its result. The merging criterion is learned through an optimization process using as reference optimal merge trees (i.e., dendrograms defined by a sequence of merges that correspond to ground truth segmentation data). Similar to [4, they factor the problem of different classification criteria across distinct scales by considering for each pair of groupings how their minimum and maximum region sizes compare to the median size over the training data.

\subsection{Sparse hierarchical segmentation}

Following our definition, any sparse hierarchical image segmentation method works by adapting an existing superpixel algorithm to recursively operate over progressively coarser image partitions. For each method, we start by describing its original non-hierarchical counterpart and follow with the actual hierarchical version.

In [15, the graph-based Image Foresting Transform (IFT) framework [26, 27] is used to frame superpixel segmentation as an iterative computation of optimum-path forests while improving a seed set with the roots of these forests. The method is called Iterative Spanning Forest (ISF), and, alongside our proposed hierarchical extension, it will be covered in more detail in Section 4.

\footnotetext{
${ }^{4}$ Not the same as gPb-owt.
} 
Also in the context of graphs, the authors in 28 propose an image segmentation algorithm based on the minimum spanning tree (MST) computation with Kruskal's algorithm. To organically identify image borders, each potential edge to be added into the MST is subjected to a predicate that compares its weight to the internal weights of the components being connected. It is fairly popular due to its simplicity, speed, and boundary adherence, but in the context of superpixels, it suffers from creating very irregular regions and not having any direct control over the resulting number of partitions. We also note that the method technically builds a dense hierarchy (each edge added to the spanning forest is a merging operation), but its intermediate states are not designed to be used as such.

Both [29] and [30] propose hierarchical strategies for image segmentation and video segmentation, respectively, taking into account the algorithm from [28] to compute the segmentation in each scale.

Instead of using Kruskal's algorithm, the Borůvka's algorithm is used in [29] to compute the MST, similar to [12]. Both 29] and [30] consider the mean pixel-level features over the region to compute edge weights in each new scale of the sparse hierarchy, but color histograms are also introduced in 30. for coarser scales, which is particularly appropriate given the high number of voxels in the video volume. Like 28, these methods inherit the lack of control over segmentation results in each new scale.

In contrast to these methods that build a sparse hierarchy using the algorithm from [28 iteratively, the authors in 31 and 32 propose an alternative method for video segmentation and image segmentation, respectively, in which the hierarchy of partitions is computed in a single-shot manner. Despite their improved segmentation results, they also do not have a fine control over the resulting number of regions in each scale.

In [33, a clustering-based superpixel method using a variation of the $k$-means algorithm is proposed, working similarly to SLIC [13]. The main difference in comparison to SLIC is how, instead of using a fixed number of iterations, they store the minimum distance from a pixel to a cluster prototype over successive iterations to force a faster convergence.

In their follow-up work [16, they improve the original method with an adaptive regularity parameter that weights local homogeneity information, and they introduce a hierarchical scheme where the clustering process is repeated using regions instead of pixels. When operating over regions, their method considers an approach to avoid setting a fixed number of clusters in advance, 
initializing each region as a cluster representative and introducing a tolerance factor in the cluster assignment function, which will lead neighboring homogeneous regions to converge into a single region. Despite its interesting theoretical properties, the method requires careful tuning of the parameters, and, in the context of superpixel segmentation, it sacrifices a finer control over the

(regions), requiring additional post-processing to build each segmentation scale.

\section{Image Graphs and the Image Foresting Transform}

In this section, we present some basic definitions which are relevant to our graph-based approach.

\subsection{Image Graphs}

Let $\mathcal{I} \subset \mathcal{P}\left(\mathcal{Z}^{2}\right) \backslash \emptyset$ be either a set of pixels or a set of superpixels (disjoint sets of simply connected pixels), where $\mathcal{P}$ indicates the power set. A 2-dimensional image is a pair $(\mathcal{I}, \mathbf{I})$, where $\mathbf{I}(t)$ assigns a finite set of scalar values to each atomic element $t$ in the image domain $\mathcal{I}$. For instance, $\mathbf{I}(t)$ may be the color of a pixel, the mean color of a superpixel, or any feature vector assigned to an atomic element $t \in \mathcal{I}$. 
For a given adjacency relation $\mathcal{A} \subset \mathcal{I} \times \mathcal{I}, G=(\mathcal{I}, \mathcal{A}, \mathbf{I})$ is said to be an image graph, where $\mathcal{I}$ is the set of nodes, $\mathcal{A}$ is the set of edges, and $\mathbf{I}(t)$ assigns scalar values to each node $t \in \mathcal{I}$. Note that we will focus on the 2-dimensional case, but these definitions, as well as our algorithms, are easily extended to the multidimensional case. The set $\mathcal{A}$ may define a 4-connected pixel graph or a Region Adjacency Graph (RAG), the latter assigning edges to each pair of superpixels in $\mathcal{I}$ containing pixels that share one edge in the pixel graph.

Given two nodes $s, t \in \mathcal{I}$, we indicate that $s$ is incident on $t$ by $(s, t) \in \mathcal{A}$. A path with terminus $t$ is defined as a sequence of nodes $\pi_{t}=\left\langle t_{1}, t_{2}, \ldots, t_{k}=t\right\rangle$, where $\left(t_{i}, t_{i+1}\right) \in \mathcal{A}$ for $1 \leq i \leq k-1$. When $k=1$, the path $\pi_{t}=\langle t\rangle$ is said to be trivial. The first node of a path is called its root and it is defined as $R\left(\pi_{t}\right)$. A path extension is represented by the operator $(\cdot)$ so that $\pi_{t_{i}} \cdot\left(t_{i}, t_{j}\right)=\left\langle t_{1}, t_{2}, \ldots, t_{i}\right\rangle \cdot\left(t_{i}, t_{j}\right)=\left\langle t_{1}, t_{2}, \ldots, t_{i}, t_{j}\right\rangle=\pi_{t_{j}}$ for any $\left(t_{i}, t_{j}\right) \in \mathcal{A}$.

A path-cost function $f\left(\pi_{t}\right)$ assigns a scalar value to any path $\pi_{t}$. Here, only additive pathcost functions with the general form $f\left(\pi_{s} \cdot(s, t)\right)=f\left(\pi_{s}\right)+g\left(R\left(\pi_{s}\right), s, t\right)$ for non-trivial paths are considered. Unlike a local edge cost, $g$ also takes into account information from the path being extended with the root $R\left(\pi_{s}\right)$. This will be used to model superpixel information in the specific path-cost functions we use (see Section 4.1).

\subsection{Image Foresting Transform (IFT)}

The Image Foresting Transform (IFT) is a variant of Dijkstra's algorithm (i.e., a dynamic programming algorithm) for more general path-cost (connectivity) functions and multiple sources [26]. Let $G=(\mathcal{I}, \mathcal{A}, \mathbf{I})$ be an image graph and $f$ a path-cost function satisfying the conditions in [27, the IFT takes $G$ and $f$ as input and outputs an optimal image partition - i.e., a minimum-cost path forest for $f$ rooted at a set $\mathcal{S} \subset \mathcal{I}$ of source nodes, called seeds. In the IFT, the seeds compete among themselves, such that each seed conquers the nodes that are more closely connected to that seed than to any other in $\mathcal{S}$.

In the iterative superpixel segmentation method described in Section 4.1, the IFT algorithm is executed multiple times over the same image graph while updating the seed set $\mathcal{S}$ to better capture image information (similar to [13, 35, but defining connected superpixels as optimum-path trees, each rooted at one seed of $\mathcal{S}$ ). Here, instead of computing a new optimum-path forest from scratch at each iteration, an optimized version of the IFT algorithm is used: the differential IFT (DIFT) algorithm for non-monotonically incremental path-cost functions [36]. After computing an initial 
optimum-path forest in the first iteration, the DIFT algorithm avoids redundant recomputation of paths whose costs do not change in the current iteration, speeding up the superpixel segmentation process.

\section{The Recursive Iterative Spanning Forest framework}

In this section, we propose our hierarchical superpixel segmentation framework, named Recursive

\footnotetext{
${ }^{5}$ In our proposed methods, we use exclusively 4-connected pixel graphs.
} 
done in various superpixel segmentation methods). When $G$ is a RAG, any pixel-based sampling strategy (including regular grid sampling) can be trivially used by converting each selected pixel to the region (node in the RAG) the pixel is part of, with the disadvantage that duplicates would have to be removed. We still use this adaptation of regular grid sampling in our proposed method as it was empirically observed that the amount of duplicates is small; an alternative that samples directly over the RAG was presented in [18].

The seed set $\mathcal{S}_{0}$ is then used alongside the path-cost function to compute an optimum-path forest with the IFT algorithm. In our proposed method we use both the mean path-cost function [15, 37. and the root path-cost function [15], which we define next. The mean path-cost function is given by

$$
\begin{aligned}
f(\langle s\rangle) & = \begin{cases}0 & \text { if } s \in \mathcal{S}, \\
+\infty & \text { otherwise. }\end{cases} \\
f\left(\pi_{s} \cdot(s, t)\right) & =f\left(\pi_{s}\right)+\underbrace{\left(\alpha\left\|\mathbf{I}(t), \mathbf{M}\left(R\left(\pi_{s}\right)\right)\right\|\right)^{\beta}}_{\text {parametric term }}+\underbrace{\|s, t\|,}_{\text {geometric term }}
\end{aligned}
$$

where $+\infty$ is an infinity cost, $\alpha \geq 0, \beta \geq 1,\|\cdot, \cdot\|$ is the Euclidean norm, and the term $\|s, t\|$ refers to the distance between the geometric positions (centroids for regions) of $s$ and $t$, respectively. $\mathbf{M}(s)$ is defined as

$$
\mathbf{M}(s)= \begin{cases}\mathbf{I}(s) & \text { if it is the first iteration, } \\ \frac{1}{|A|} \sum_{t \in A} \mathbf{I}(t) & \text { otherwise, }\end{cases}
$$

where $A=\left\{t \in \mathcal{I} \mid R\left(\pi_{t}^{\prime}\right)=R\left(\pi_{s}^{\prime}\right)\right\}$, with $\pi^{\prime}$ indicating the optimum path obtained in the previous iteration. In other words, $\mathbf{M}\left(R\left(\pi_{s}\right)\right)$ is the mean value of $\mathbf{I}$ within the optimum-path tree covering the seed $R\left(\pi_{s}\right)$ in the previous iteration, or the color of the seed itself in the first iteration.

Similarly, the root path-cost function is defined as

$$
\begin{aligned}
f(\langle s\rangle) & = \begin{cases}0 & \text { if } s \in \mathcal{S} \\
+\infty & \text { otherwise. }\end{cases} \\
f\left(\pi_{s} \cdot(s, t)\right) & =f\left(\pi_{s}\right)+\left(\alpha\left\|\mathbf{I}(t), \mathbf{I}\left(R\left(\pi_{s}\right)\right)\right\|\right)^{\beta}+\|s, t\| .
\end{aligned}
$$

For both path-cost functions, the cost of each path extension considers a mixed distance on the parametric and geometric spaces. The parametric term is responsible for measuring the similarity between nodes according to the values assigned by $\mathbf{I}$, with each function differing in what information is used to model border evidence. The geometric term, identical in both functions, acts as a 
regularization term that ensures smooth borders in homogeneous areas of the image and constrains errors over weak borders.

The two parameters, $\alpha$ and $\beta$, control the regularity of the resulting partition. The value of $\alpha$ controls the relative difference between the parametric and geometric terms, with higher values resulting in less regular regions. This value depends on the choice of parametric space, and the specific values used in our proposed method are defined in the experimental analysis. Meanwhile, $\beta$ controls the relative weight of each edge added to the path: $\beta=1$ means each edge has equal weight, $\beta \rightarrow \infty$ makes $f$ work like a max function (i.e., path-cost is the cost of the edge with maximum weight), and values in-between make $f$ work like a soft-max function. Generally, lower values give more regular results. In our method, a fixed $\beta=12$ is considered, as done in [15].

As noted in Section 3.2, the resulting optimum-path forest from the IFT computation is already a partition of the image into regions. At this point, there are two options: taking the result as the final superpixel segmentation based on some convergence criteria (typically replaced by a fixed number of iterations in practice [13, 15], $T=10$ iterations in our proposed method), or using it as input for the seed recomputation procedure to build an improved seed set. In the latter case, a new iteration of the IFT computation occurs with the improved seed set, and the process is repeated until the former option is selected to end the algorithm.

We consider the same seed recomputation procedure from [15, which builds the improved seed set $\mathcal{S}_{i+1}$ as follows. For each seed $s \in \mathcal{S}_{i}$, a new candidate seed $s^{\prime} \in \mathcal{I}$ is computed inside the region $\mathcal{R}$ spanned by $s$ (i.e., the tree rooted in $s$ ). When using the mean and root path-cost functions, $s^{\prime}$ is the node closest to the centroid of $\mathcal{R}$ and the one with color most similar to the mean color of $\mathcal{R}$, respectively. Then, $s^{\prime}$ is added to $\mathcal{S}_{i+1}$ if its distance to $s$ in either the parametric or geometric space is above a dynamic threshold based on $\mathcal{R}$. Otherwise, $s$ is added to $\mathcal{S}_{i+1}$.

The full operation of ISF is summarized in Figure 3 . We also present the corresponding algorithm in Figure 4. In our proposed method, the following ISF components are considered. The seed sampling strategy (Line 1) is exclusively regular grid sampling. The path-cost function (Line 3) is either the mean path-cost function (Equation 2) or the root path-cost function (Equation 4). The seed recomputation procedure (Line 4) selects the node closest to the centroid or the mean color of each region. Lastly, the convergence criteria (Line 5) is simplified to a fixed number of $T=10$ iterations. For an in-depth description including the IFT algorithm and its sub-components, see [18]. 


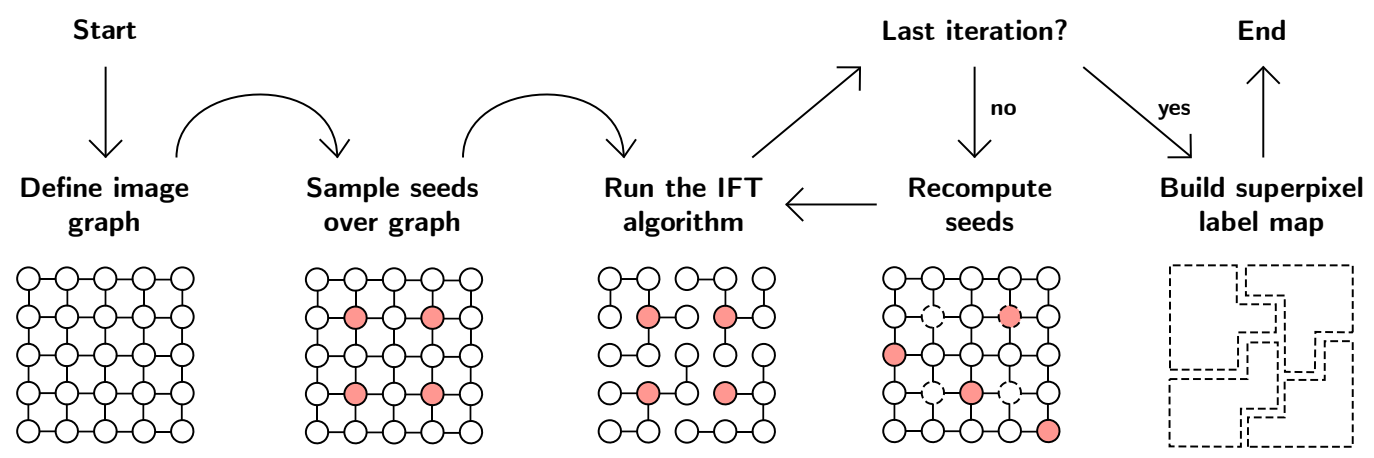

Figure 3: Overview of the major steps of an ISF method. The steps are illustrated with a 4-connected pixel graph as the image graph. In each step, the seeds are highlighted, and, for the seed recomputation part, former seeds are indicated with a dotted circle.

Input: Image graph $G=(\mathcal{I}, \mathcal{A}, \mathbf{I})$ and target number of regions $n$.

Output: Labeled image $L$ with $n$ regions.

1: Compute $\mathcal{S}$ with the seed sampling strategy

2: repeat

3: $\quad$ Run IFT with $G, \mathcal{S}$ and a path-cost function $f$ to obtain a labeled image $L$

4: $\quad$ Update $\mathcal{S}$ with the seed recomputation procedure

5: until $L$ converges

6: return $L$

Figure 4: ISF algorithm.

The definition of a RISF method consists of defining multiple ISF methods with the added restriction that the target number of regions must be decreasing. The algorithm to build the hierarchy of image segmentations with RISF is shown in Figure 5, noting that it matches the previously 
Input: Image $(\mathcal{I}, \mathbf{I})$, sequence $\left\langle\left(I S F_{1}, n_{1}\right),\left(I S F_{2}, n_{2}\right), \ldots,\left(I S F_{K}, n_{K}\right)\right\rangle$ with an ISF method $I S F_{k}$ and number $n_{k}$ of regions per observation scale, where $n_{k}>n_{k+1}, k=1,2, \ldots, K-1$ and $n_{K}$ is the desired number of regions in the coarsest level.

Output: Sparse hierarchy $\mathcal{H}$ with $K$ observation scales.

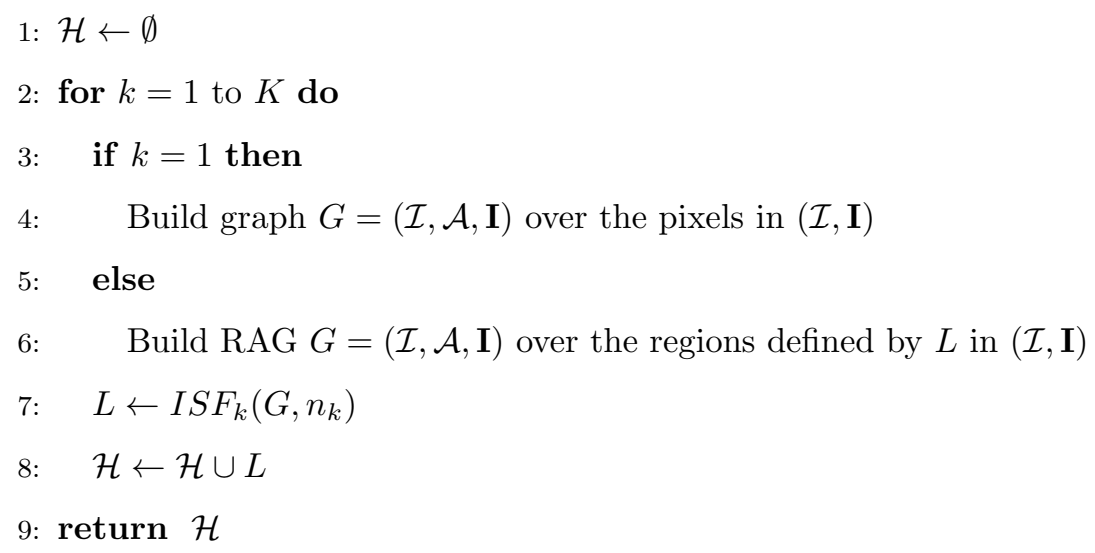

Figure 5: RISF algorithm.

presented high-level diagram in Figure 1. For the input, we recall that an ISF method refers to a predefined selection of a seed sampling strategy, a path-cost function, a seed recomputation procedure, and any other parameters associated with the chosen components (see Section 4.1). Each iteration of the loop in Lines 2-8 builds a new image segmentation with the corresponding number of regions $n_{k}$ until the sparse hierarchy $\mathcal{H}$ is complete, with the RAG definition in Line 6 characterizing our adaptation of ISF for the RISF framework.

As each image segmentation step groups superpixels from the previous one (or pixels in the first segmentation), there is an order relation between elements of each scale and thus making RISF's output a true hierarchy. While ISF (the original method over pixels) and most other superpixel segmentation algorithms can have their parameters changed to create multiple image segmentations with different levels of coarseness, generally this approach would not give a true hierarchy because the resulting set of segmentations would violate either the causality principle - that the contours present in a given observation scale should also be present in any finer scale - or the location principle - that the contours should be stable, not moving across different observation scales [38. This is illustrated in Figure 6, where we show the relationship between different observation scales from RISF and independent ISF methods. 


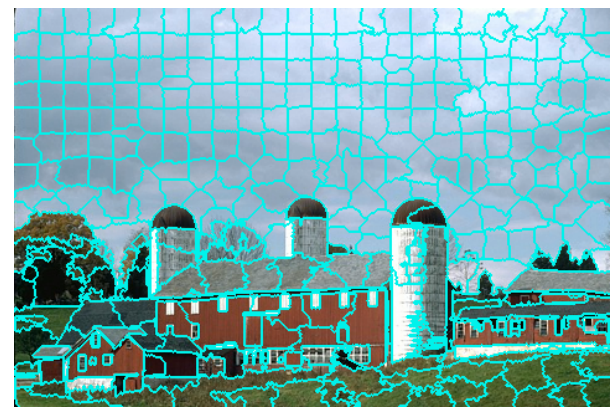

(a) $\operatorname{ISF}(N=400)$

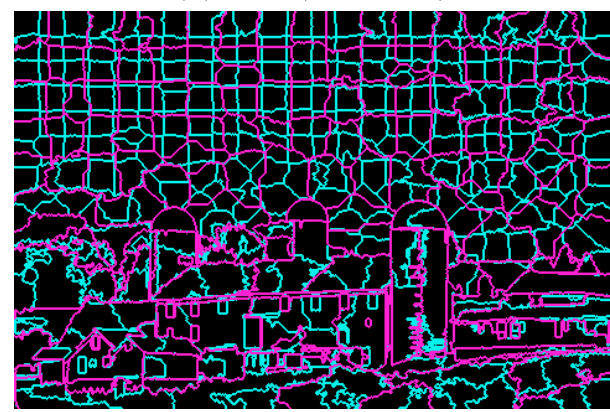

(c) Overlaid contours

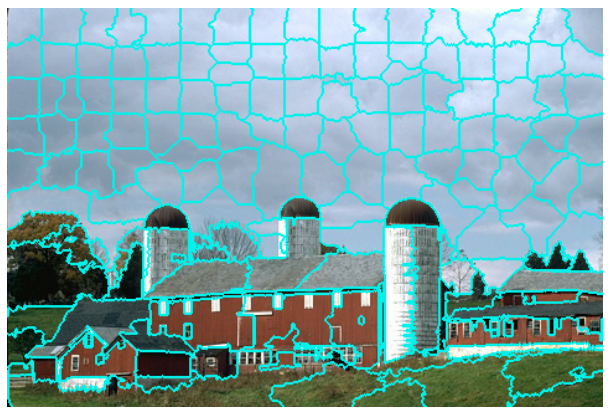

(b) $\operatorname{ISF}(N=200)$

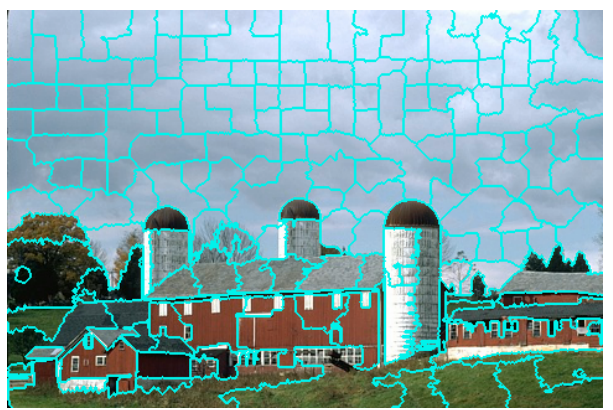

(d) $\operatorname{Ours}(N=200)$

Figure 6: Comparison of non-hierarchical and hierarchical image segmentation with the Iterative Spanning Forest framework [15] and our proposed hierarchical extension. Like most superpixel segmentation methods (i.e., nonhierarchical ones), executing the original algorithm over different scales (a-b) results in contours that violate the causality and location principles [38] as shown in (c). Meanwhile, our proposed version (d) groups the regions in (a) to generate a proper hierarchical segmentation, as seen by the fact the contours in (d) are a subset of the ones in (a).

It is also worth noting that building each level of the hierarchy of superpixels using pixels as image primitives would increase the overall processing time in proportion to the number of hierarchy levels. In contrast, RISF produces the entire hierarchy faster than a single ISF segmentation, as we later show in the experimental analysis.

Following from Section 2.2 , the concept of recursively applying a graph-based superpixel segmentation algorithm to generate a sparse hierarchy was explored by the authors in [29] and [30], using the graph-based segmentation from [28] in images and videos, respectively. As compared to the existing graph-based approaches, RISF introduces some advantages including: an underlying segmentation method with state-of-the-art boundary adherence results, the whole process being faster than generating the coarsest scale directly due to particular properties of ISF, and direct 

Lines 6-9, all other edges containing either node from the selected edge are removed from $\mathcal{Q}$. In Line 10, the actual merge occurs, performing the previously described graph update. In Lines 11 
and 12 , the edges incident on the new merged node are added into $\mathcal{Q}$. Lastly, in Line 13, we store the current segmentation defined by the updated image graph. This algorithm builds the hierarchy as a stack of partitions from each merge, but we note that the hierarchy can also be stored with a more compact representation, such as an ultrametric contour map (UCM).

Input: $\operatorname{Graph} G=(\mathcal{I}, \mathcal{A}, \mathbf{I})$

Output: Dense hierarchy of superpixel segmentations $\mathcal{D}$

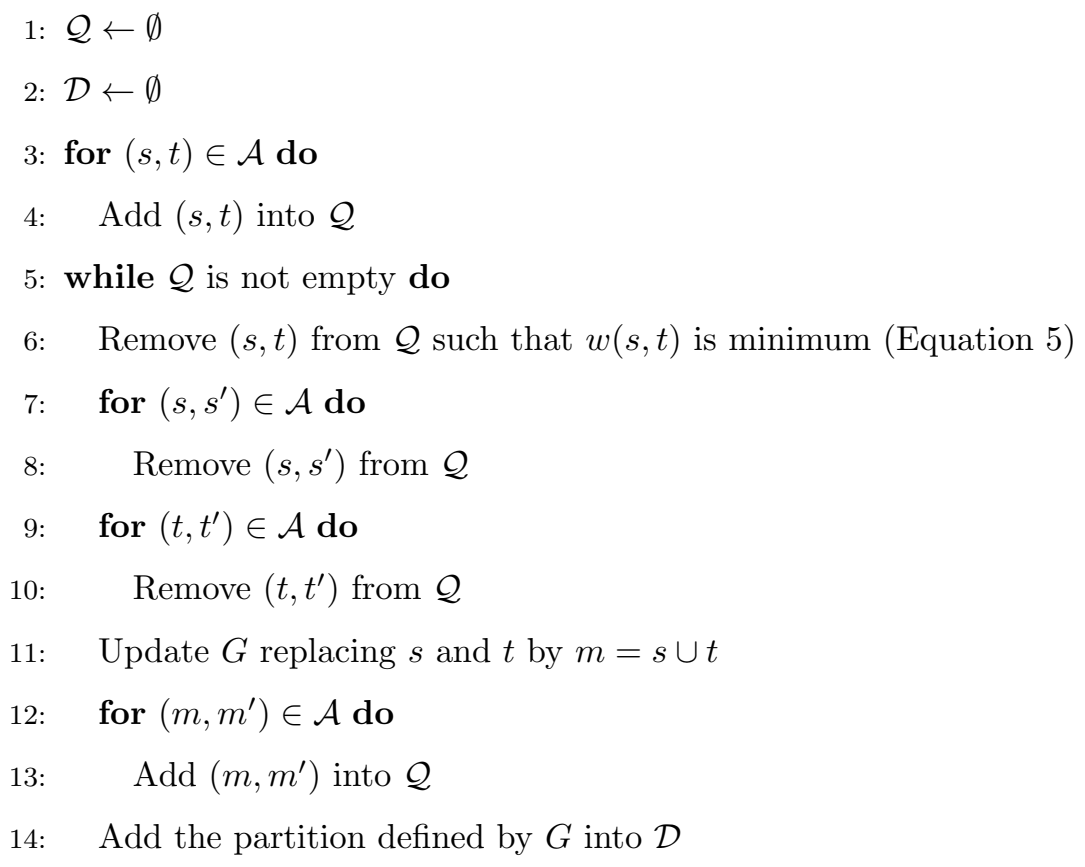

Figure 7: Mid-level Region Merge algorithm.

Unlike [11, we do not have a sub-modular function to perform heap updates in linear time, which is compensated by the fact we already use a simplified RAG as a starting point. Assuming a binary heap is used, the overall complexity of operating the heap is $O(n \log n)$, where $n$ is the initial number of regions $|\mathcal{I}|$, and the number of edges is comparable to $n$ (note that $G$ is planar).

To efficiently represent the progressively coarser RAG using its initial state as a base, a forest of disjoint sets structure with union-find operations can be used [39]. Let $s \cup t$ be the node obtained merging nodes $s$ and $t$, we show next how to compute its necessary information after each update in constant time. Assuming $\mathbf{I}$ is defined as the mean value of some pixel-level property (e.g., color) 
over a node's corresponding region, we have

$$
\mathbf{I}(s \cup t)=\frac{\mathbf{I}(s) \cdot|s|+\mathbf{I}(t) \cdot|t|}{|s|+|t|},
$$

which can be computed in constant time. Likewise, $|s \cup t|=|s|+|t|$ and $(u, s \cup t) \in \mathcal{A}$ if $(u \neq$ $t \wedge(u, s) \in \mathcal{A}) \vee(u \neq s \wedge(u, t) \in \mathcal{A})$, the latter operation assuming $G$ is implemented with adjacency lists and that the number of neighbors for each node is insignificant, as compared to the number of nodes. Thus, analogous to Kruskal's minimum spanning tree algorithm [40], the complexity of using the forest of disjoint sets structure is also $O(n \log n)$, making the overall complexity of our region-merging algorithm $O(n \log n)$.

\section{Experimental analysis}

Here we present an empirical evaluation of different hierarchical segmentation methods against the state-of-the-art in superpixel segmentation. For hierarchical methods, we focus on those generating hierarchies of superpixels.

\subsection{Datasets}

We consider three natural image datasets in our experiments. They are the BSDS500 99, the MSRC 41, and the Birds $42^{6}$ datasets. A short description of each one is presented next.

The BSDS500 is a standard image segmentation benchmark and particularly prominent in the superpixel literature. It is composed of 500 images, each with $481 \times 321$ resolution and multiple segmentation ground truths indicating the borders perceived by different subjects. Only the dataset's 200 test images were considered in the reported results, with the other 300 training and validation images being exclusively used for parameter tuning (including for experiments performed on the next two datasets).

The MSRC dataset contains 591 images with segmentations of 23 different object classes. Its images have $320 \times 213$ resolution, and we use the cleaned up and more accurate ground truths provided by the authors in [43]. We also remove 8 samples that contain no borders (the entire image is a single class).

\footnotetext{
${ }^{6}$ Extended version including 100 extra bird images available on http://www.vision.ime.usp.br/ pmiranda/ downloads/COIFT/COIFT.html
} 
The Birds dataset has 150 public domain images of birds that are challenging to segment due to their thin and elongated parts. Each image has a resolution up to $640 \times 640$ and a manually generated ground truth segmentation of a single bird in the scene.

\subsection{Experimental settings}

As described in Section 4 , both ISF and RISF are frameworks and require a specification of each component to define an actual segmentation method to be evaluated.

In the results, we refer to ISF as the original algorithm [15] working over the 4-connected pixel graph, performing grid sampling, and using the mean path-cost function (Equation 2) alongside the seed recomputation which selects the centroid of a region as seed (see Section 4.1). In [15, different ISF methods were considered to optimize the results on different datasets to show the flexibility of the framework. However, for simplicity, and because the observed difference in the segmentation results is minor, here we consider only the ISF method we just described (ISF-GRID-MEAN in their work).

Likewise, the method referred to as RISF in the results uses the aforementioned ISF method to build its first segmentation with $N=6400$ regions, and then, for the following scales, uses a different ISF method which is defined over RAGs, performs grid sampling, and uses the root pathcost function (Equation 4 ) alongside the seed recomputation selecting the node with closest color to the region mean as seed; each new scale reduces the number of regions by a factor of 1.5 until $N \approx 50$ regions, resulting in a total of 13 observation scales.

We also consider the variant RISF-old based on the experimental settings used in 18; its difference to RISF is that it uses the same path-cost function and seed recomputation procedure as ISF in all scales. Additionally, the factor of reduction after each scale is varied to evaluate the performance of RISF-old when building hierarchies with different number of observation scales. Everything else defined for RISF next is also valid for RISF-old.

Both the mean and root path-cost functions have two parameters: $\alpha$, the main regularization parameter (higher values give less regular superpixels), and $\beta$, a secondary regularization parameter balancing the relative weight of individual edges. Based on an optimization over the BSDS500 training and validation sets, both ISF and RISF use $\alpha=0.5$ and $\beta=12$, except in the first segmentation of RISF where we use $\alpha=0.2$ to make the algorithm faster (fewer updates in the DIFT). At such a fine initial scale, this difference does not impact the quality of the segmentation 
negatively. Efficiency is also the reason for using the mean path-cost function only in the first segmentation of RISF.

Following from Section 4.3 , the mid-level region merge algorithm has no parameters but requires an initial over-segmentation $\sqrt{7}$ For that we use one of the generated RISF scales with $N \approx 600$ regions as the starting point, and we refer to this combination of RISF and the merging algorithm as RISF-merge in the results. The choice of RISF is motivated by a separate set of experiments included in the supplementary material where we evaluate the combination of the mid-level region merge algorithm with the other segmentation methods included here.

For both ISF and the first segmentation of RISF, we use the publicly available implementation of the method over pixels 8 . For RISF's operation over RAGs and the mid-level region merge algorithm, our implementation is available in the supplementary material.

In all RAGs of our proposed methods (RISF and mid-level region merge), we assume that $\mathbf{I}(t)$ is the mean pixel color (CIELAB color space) over the region represented by node $t$. Likewise, in any ISF component that originally considered pixel coordinates, we used the region centroid instead.

Based on availability of a public implementation, the other methods we cover in our experiments to 4-neighbors, which we argue is more due to limitations in how BR is defined than an actual improvement in boundary adherence.

\footnotetext{
${ }^{7}$ This method can be applied directly to the pixel graph, but, according to empirical analysis, its performance degrades if the starting point is too fine.

$\varepsilon$ https://www.ic.unicamp.br/ afalcao/downloads.html

'https://www2.eecs.berkeley.edu/Research/Projects/CS/vision/grouping/resources.html\#algorithms

10 https://www2.eecs.berkeley.edu/Research/Projects/CS/vision/grouping/mcg/

11 https://github.com/mingyuliutw/EntropyRateSuperpixel

12 https://github.com/semiquark1/boruvka-superpixel

13 https://jschenthu.weebly.com/projects.html

14 https://sites.google.com/site/pierrebuyssens/code/graph-based-oversegmentation

${ }^{15}$ https://www.epfl.ch/labs/ivrl/research/research-current/research-superpixels/
} 
Based on Section 2 the categorization of the covered methods is as follows. The dense hierarchical segmentation methods are gPb-owt-ucm, MCG-fast, MCG-acc, RISF-merge, ERS, and SH, with only the last three being proposed with superpixel segmentation in mind. The sparse hierarchical segmentation representative is RISF. Lastly, as some reference state-of-the-art non-hierarchical superpixel segmentation methods, there are ISF, LSC, ERGC, and SLIC.

\subsection{Evaluation measures}

Similar to 4, to evaluate the actual partitions we make a distinction for segmentations with more than 50 regions as the superpixel regime, and less than 50 regions as the large segment regime, using the appropriate measures for each case. Additionally, we look at the processing time each method takes to generate the entire hierarchy (or a single arbitrary scale for non-hierarchical methods).

As the name suggests, the superpixel regime covers segmentations typically associated with superpixel segmentation methods. As noted in various works in the superpixel literature [22, 13, boundary adherence is the primary segmentation property to be considered in this regime. In this context, over-segmentation is assumed to happen a priori, and, as a result, typical measures do not penalize it directly. Here we consider two standard boundary adherence measures: boundary recall (BR, as defined in [13]) and undersegmentation error (UE, as defined in [45]). Each measure is evaluated for a range of segmentation scales (i.e., number of superpixels).

The large segment regime, on the other hand, is covered by methods aiming to capture highlevel information and achieve a segmentation as close as possible to the ground truth. Similar to other works focused on this regime (e.g., 4, 24]), we consider boundary and region-based measures appropriate for this regime. Those measures are boundary F-score (BF, analogous to BR but also taking into account boundary precision information), segmentation covering (shortened to Cover in the results, using definition from [9]), variation of information (VOI) [4] and probabilistic Rand index (PRI, assuming uniform probability distribution across ground truths) [47. Following [9], we consider for each measure the optimized value for a fixed scale across the entire dataset (ODS) and the best aggregated value optimizing the scale individually for each sample in the dataset (OIS).

Despite not being appropriate for superpixel methods in general, the large segment regime serves as a way to evaluate how much high-level information is captured in the coarser scales of a dense hierarchical segmentation method, even if the method was designed to generate superpixels. Additionally, comparing the performance of superpixel-oriented methods against those dedicated 
to this mode of operation will serve as a way to indicate that our mid-level region merge algorithm is closer to higher-level segmentation methods.

We emphasize that superpixel-oriented methods are not expected to outperform methods focused on the large segment regime in terms of segmentation accuracy; this is a result of their heavier emphasis on regularity and concessions in favor of performance. Thus, we explicitly separate both categories, superpixel and non-superpixel, when presenting the results of the large segment regime. Additionally, in the results we also discuss how the large segment regime measures can be misleading

\subsection{Results}

In Table 1 we compare the boundary adherence performance of the current version of RISF and the one presented in [18] (RISF-old) in a coarse scale of the superpixel regime, showcasing that the boundary adherence performance of RISF-old is only comparable to RISF in a trivial hierarchy with 2 scales. Even adding a single intermediate scale significantly impairs the performance of RISF-old, making it unsuitable in the context of this work where the hierarchical representation in itself is important. Thus, we only cover RISF in the remainder of this section.

\begin{tabular}{c|c|c} 
Method & BR & UE \\
\hline RISF (11 scales) & 0.774 & 0.181 \\
RISF-old (11 scales) & 0.747 & 0.257 \\
RISF-old (8 scales) & 0.740 & 0.247 \\
RISF-old (4 scales) & 0.757 & 0.201 \\
RISF-old (3 scales) & 0.753 & 0.210 \\
RISF-old (2 scales) & 0.771 & 0.171
\end{tabular}

Table 1: Comparison of RISF and RISF-old on the BSDS500 dataset for $N \approx 100$ regions. The indicated number of scales is the total generated to reach $N \approx 100$, starting from $N=6400$ and using a constant reduction factor.

The average processing time for each of the evaluated methods to generate either a full hierarchy or some reference observation scales on the BSDS500 dataset is presented in Table 2, noting that all experiments were run on an Intel i7-7700 3.60GHz. As predicted, superpixel-oriented methods are significantly faster than the evaluated non-superpixel methods, noting that MCG-fast speed is already amongst the state-of-the-art methods in the related literature, but for the non-hierarchical 
methods that advantage is restricted to the generation of individual observation scales. Across the superpixel-oriented methods, $\mathrm{SH}$ is the fastest one with both of our proposed methods following next.

Here we highlight the first benefit of our proposed RISF method in comparison to the original ISF one. It manages to be faster despite generating an entire hierarchy. In RISF, the first iteration over pixels benefits from ISF's property of being faster when targeting a larger number of superpixels (as first noted in [15, which happens due to DIFT being able to perform more localized updates), while the following iterations benefit from the reduced number of elements in the RAG. The difference between RISF and RISF-merge is due to the fixed time overhead for each extra scale added in the sparse hierarchical segmentation loop, while the merging algorithm can generate the remaining dense hierarchy directly and fast (within a millisecond).

\begin{tabular}{c|cccc} 
& \multicolumn{5}{|c}{ Time $(\mathrm{ms})$} \\
Method & Full Hierarchy & Coarse $(N \approx 50)$ & Mid $(N \approx 600)$ & Fine $(N \approx 6400)$ \\
\hline RISF & 110.6 & 110.6 & 97.1 & 62.0 \\
RISF-merge & 97.3 & 97.3 & 97.1 & 62.0 \\
ISF & 2317.2 & 226.5 & 189.9 & 94.5 \\
gPb-owt-ucm & 80336.9 & - & - & - \\
MCG-fast & 1499.6 & - & - & - \\
MCG-accurate & 9435.8 & - & - & - \\
ERS & 526.1 & 526.1 & 481.7 & 420.7 \\
SH & 40.7 & 40.7 & 40.6 & 40.5 \\
LSC $^{\star}$ & 3145.3 & 228.6 & 243.8 & 234.2 \\
ERGC $^{\star}$ & - & 220.1 & 1246.9 & - \\
SLIC $^{\star}$ & 1333.5 & 96.8 & 103.2 & 105.8
\end{tabular}

Table 2: Average processing time to compute the entire segmentation hierarchy and some reference scales for each evaluated method on the BSDS500. For the reference scales, we indicate the time taken to reach the corresponding number of regions when starting from pixels. For non-hierarchical methods (indicated by the $\star$ ), the full hierarchy considers the time to generate the same 13 hierarchy levels covered by RISF. Both gPb-owt-ucm and MCG methods output an ultrametric contour map representing the entire hierarchy, so we do not include the time for individual scales on those. ERGC crashed on the 4 finest hierarchy levels. 
The superpixel regime results are presented in Figures 8,9 and 10. From the non-superpixel

A visual comparison of each superpixel segmentation method in this regime is presented in

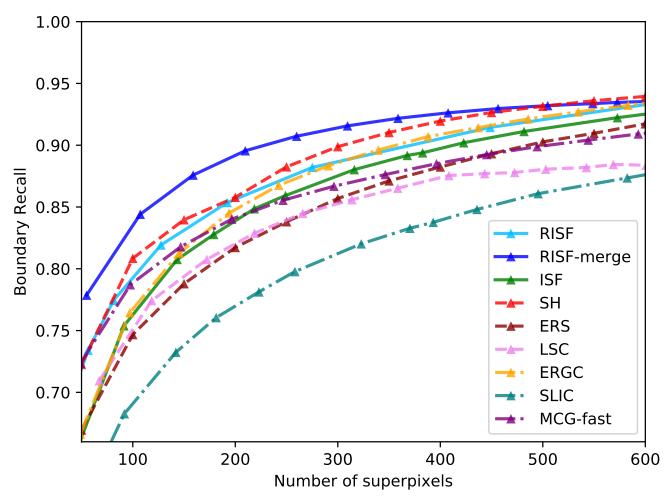

(a)

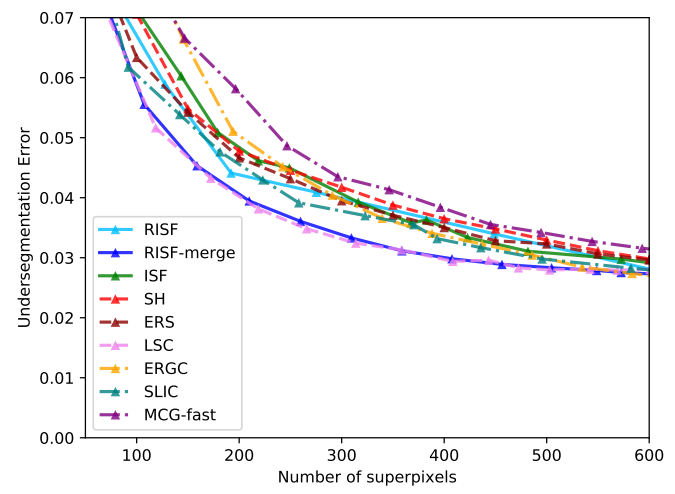

(b)

Figure 8: Boundary adherence measures for the superpixel regime on the BSDS500 dataset. 
Figure 11. Here we focus on how regular the partitions of each method look. Compared to ISF, RISF ends up with more irregular shapes as the regularization term only considers the centroid of each region. MCG-fast generates a lot of spurious regions around object borders, and SH produces very irregular borders even in homogeneous regions. Additionally, ISF, RISF, LSC, and SLIC have superpixels aligned close to a grid - result of their underlying grid sampling - a property exploited by some higher level methods using superpixels and which is notably missing from dense hierarchical methods (ERGC as well, but that is due to the alternate seed sampling strategy used

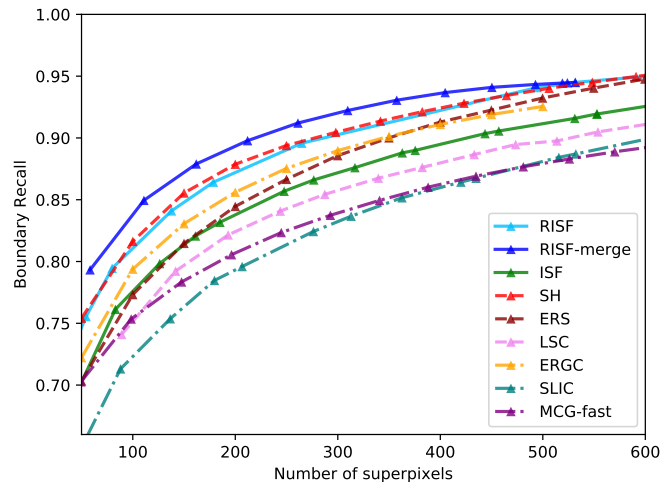

(a)

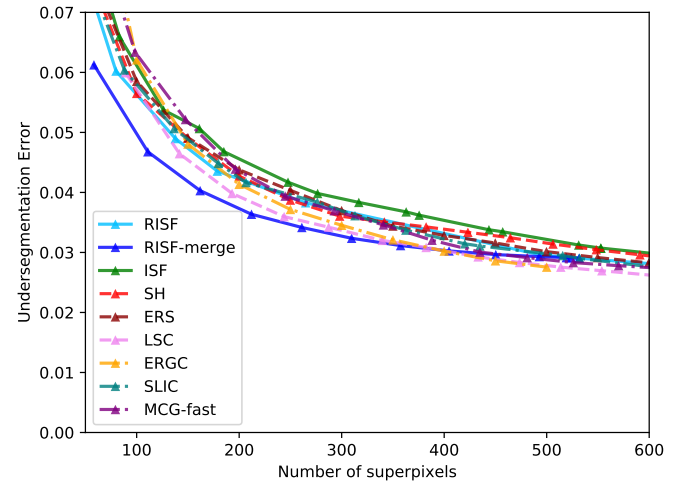

(b)

Figure 9: Boundary adherence measures for the superpixel regime on the MSRC dataset.

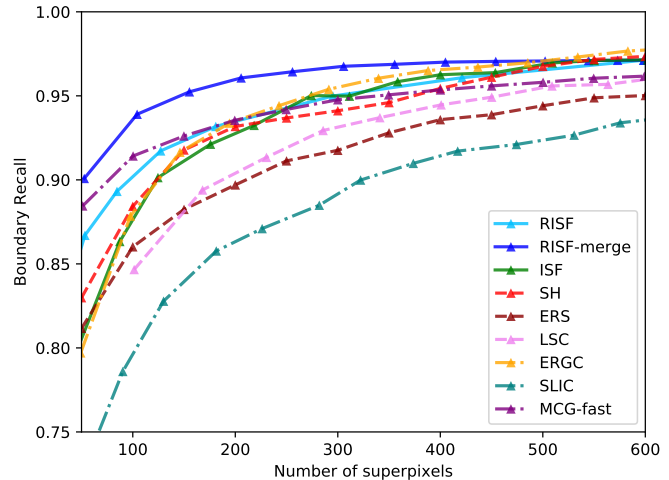

(a)

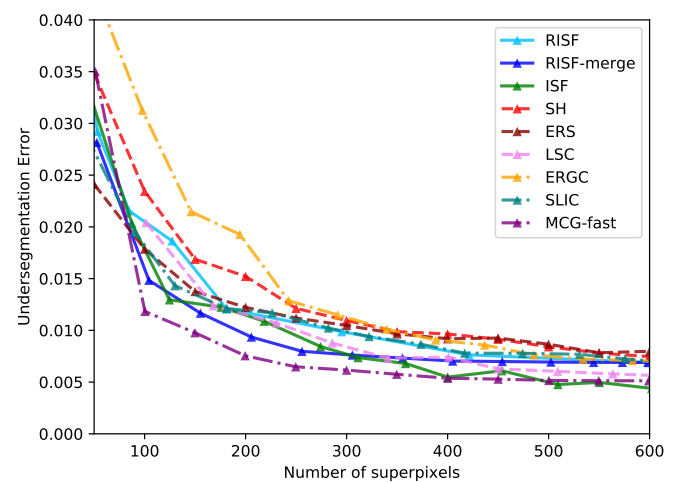

(b)

Figure 10: Boundary adherence measures for the superpixel regime on the Birds dataset. 


\begin{tabular}{|c|c|c|c|c|c|c|c|c|c|c|c|c|}
\hline & \multicolumn{4}{|c|}{ BSDS500 } & \multicolumn{4}{|c|}{ MSRC } & \multicolumn{4}{|c|}{ Birds } \\
\hline & $\mathrm{BF}$ & Cover & VOI & PRI & $\mathrm{BF}$ & Cover & VOI & PRI & $\mathrm{BF}$ & Cover & VOI & PRI \\
\hline gPb-owt-ucm & $0.52^{22}$ & $0.59^{12}$ & $1.19^{12}$ & $0.83^{41}$ & - & - & - & - & - & - & - & - \\
\hline MCG-fast & $0.55^{23}$ & $0.60^{09}$ & $1.15^{11}$ & $0.83^{26}$ & $0.45^{08}$ & $0.67^{03}$ & $0.86^{03}$ & $0.77^{08}$ & $0.56^{09}$ & $0.77^{02}$ & $0.51^{02}$ & $0.76^{02}$ \\
\hline MCG-acc & $0.56^{28}$ & $0.61^{11}$ & $1.12^{11}$ & $\mathbf{0 . 8 4 ^ { 3 0 }}$ & $0.46^{07}$ & $0.68^{03}$ & $\mathbf{0 . 8 4 ^ { 0 3 }}$ & $\mathbf{0 . 7 8 ^ { 0 9 }}$ & $\mathbf{0 . 5 7 ^ { 0 8 }}$ & $0.78^{02}$ & $0.48^{02}$ & $0.77^{02}$ \\
\hline RISF-merge & $\mathbf{0 . 3 7 ^ { 0 6 }}$ & $\mathbf{0 . 5 1 ^ { 0 3 }}$ & $1.48^{04}$ & $0.76^{10}$ & $\mathbf{0 . 3 5 ^ { 0 4 }}$ & $0.62^{02}$ & $0.98^{02}$ & $\mathbf{0 . 7 1} 1^{04}$ & $0.36^{03}$ & $\mathbf{0 . 6 3} 3^{02}$ & $\mathbf{0 . 7 2 ^ { 0 2 }}$ & $\mathbf{0 . 6 4 ^ { 0 2 }}$ \\
\hline ERS & $0.36^{11}$ & $0.48^{03}$ & $1.51^{04}$ & $0.76^{08}$ & $0.31^{04}$ & $0.53^{02}$ & $1.17^{02}$ & $0.66^{04}$ & $0.38^{03}$ & $0.56^{02}$ & $0.82^{02}$ & $0.59^{02}$ \\
\hline $\mathrm{SH}$ & $0.33^{06}$ & $0.47^{02}$ & $1.57^{04}$ & $0.75^{21}$ & $0.29^{04}$ & $0.59^{02}$ & $1.04^{02}$ & $0.66^{04}$ & $0.28^{04}$ & $0.64^{02}$ & $0.73^{02}$ & $0.64^{02}$ \\
\hline
\end{tabular}

Table 3: Large segment regime results of all evaluated dense hierarchical methods for each dataset and a fixed segmentation scale (ODS). We separate the methods into two sets - non-superpixel (top) and superpixel (bottom) — and highlight the best performing method in each set. Superscript indicates the optimal scale's number of regions for the respective evaluation measure.

in the evaluated version of the method instead of an inherent limitation).

The ODS and OIS large segment regime results are presented in Tables 3 and 4 respectively. As gPb-owt-ucm failed to segment some images on the MSRC and Birds datasets, we only include its results on the BSDS500 dataset. As expected, the non-superpixel methods do outperform the superpixel methods by a considerable margin, and the gPb-owt-ucm, MCG-fast, and MCG-acc results match those reported in [24]. Between the dense hierarchical superpixel methods, RISFmerge is either the best or tied for best in all measures, reinforcing that its performance capturing high-level information is also better than the competing methods.

In Figure 12 we illustrate the difference between superpixel-oriented methods and those dedicated to the large segment regime using RISF-merge and MCG-fast as reference. The measured BF for (e) and (f) are 0.232 and 0.315 , respectively, and we also note that the OIS number of regions based on Cover and VOI are even lower for both methods. First, we mention the limitation of optimizing for the typical large segment regime measures, observing that the selected scales can hardly be considered usable despite both (b) and (c) presenting most of the relevant borders. Second, we highlight how the typical superpixel segmentation behavior of adding more borders in oversegmented homogeneous regions leads to a harsher penalization of segmentation errors in those measures when compared to a method like MCG-fast, which is biased towards border detection even in finer scales.

Finally, we note that the supplementary material expands on the qualitative evaluation for 


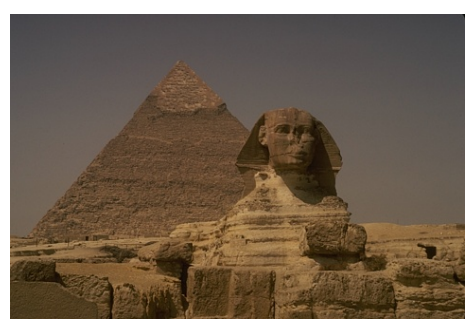

(a) Original image

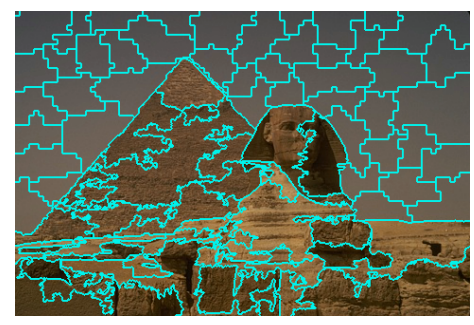

(b) RISF

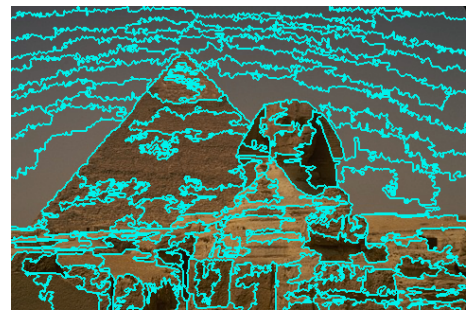

(e) $\mathrm{SH}$

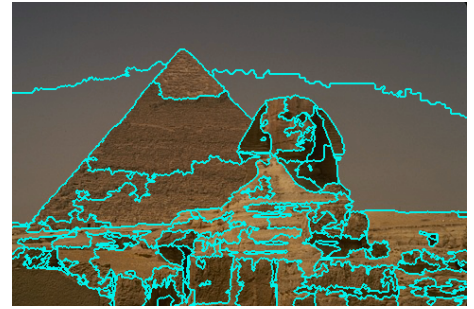

(h) ERGC

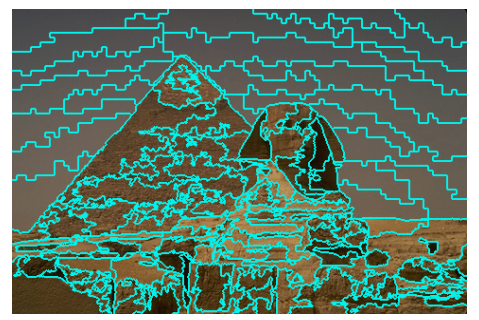

(c) RISF-merge

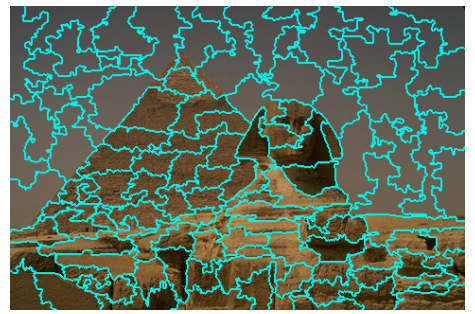

(f) ERS

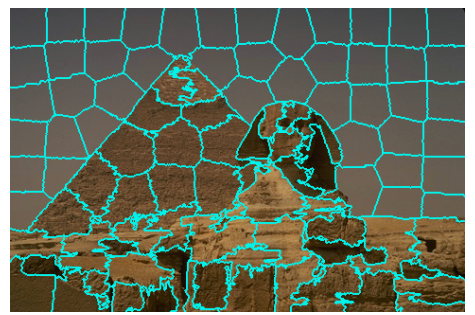

(i) SLIC

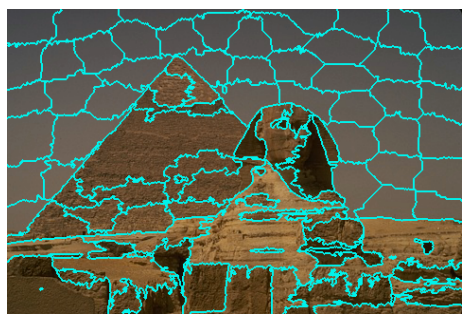

(d) ISF

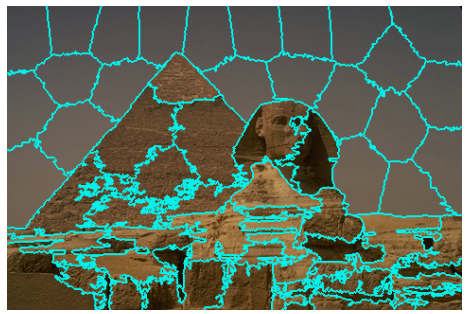

(g) LSC

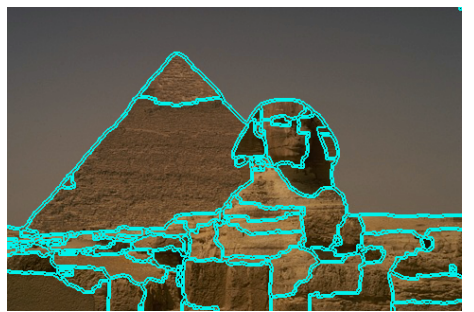

(j) MCG-fast

Figure 11: Segmentation examples from each evaluated method in the superpixel regime with $N \approx 100$ superpixels. 


\begin{tabular}{|c|c|c|c|c|c|c|c|c|c|c|c|c|}
\hline & \multicolumn{4}{|c|}{ BSDS500 } & \multicolumn{4}{|c|}{ MSRC } & \multicolumn{4}{|c|}{ Birds } \\
\hline & $\mathrm{BF}$ & Cover & VOI & PRI & $\mathrm{BF}$ & Cover & VOI & PRI & $\mathrm{BF}$ & Cover & VOI & PRI \\
\hline gPb-owt-ucm & $0.56^{22}$ & $0.66^{13}$ & $1.01^{15}$ & $0.85^{29}$ & - & - & - & - & - & - & - & - \\
\hline MCG-fast & $0.59^{22}$ & $0.67^{12}$ & $0.98^{13}$ & $0.86^{25}$ & $0.52^{11}$ & $0.74^{05}$ & $0.73^{05}$ & $0.84^{12}$ & $0.63^{09}$ & $0.79^{03}$ & $0.47^{03}$ & $0.79^{04}$ \\
\hline MCG-acc & $0.60^{25}$ & $0.68^{12}$ & $0.96^{14}$ & $0.86^{27}$ & $0.53^{12}$ & $0.75^{05}$ & $\mathbf{0 . 7 0 ^ { 0 5 }}$ & $0.85^{13}$ & $0.65^{10}$ & $0.80^{03}$ & $0.44^{03}$ & $\mathbf{0 . 8 0 ^ { 0 4 }}$ \\
\hline RISF-merge & $0.42^{10}$ & $\mathbf{0 . 5 8 ^ { 0 5 }}$ & $1.28^{06}$ & $\mathbf{0 . 8 1} 1^{13}$ & $0.43^{11}$ & $0.69^{04}$ & $\mathbf{0 . 8 6 ^ { 0 4 }}$ & $0.80^{07}$ & $\mathbf{0 . 4 5} 5^{04}$ & $\mathbf{0 . 6 4 ^ { 0 2 }}$ & $\mathbf{0 . 7 0 ^ { 0 2 }}$ & $0.66^{03}$ \\
\hline ERS & $0.41^{12}$ & $0.56^{04}$ & $1.31^{05}$ & $0.80^{09}$ & $0.38^{08}$ & $0.59^{03}$ & $1.06^{03}$ & $0.73^{05}$ & $0.45^{04}$ & $0.56^{02}$ & $0.81^{02}$ & $0.60^{02}$ \\
\hline $\mathrm{SH}$ & $0.38^{11}$ & $0.54^{05}$ & $1.38^{06}$ & $0.79^{16}$ & $0.36^{08}$ & $0.64^{03}$ & $0.95^{03}$ & $0.76^{07}$ & $0.35^{04}$ & $0.64^{02}$ & $0.71^{02}$ & $0.66^{03}$ \\
\hline
\end{tabular}

Table 4: Large segment regime results of all evaluated dense hierarchical methods for each dataset and using each sample's best segmentation scale (OIS). We separate the methods into two sets - non-superpixel (top) and superpixel (bottom) — and highlight the best performing method in each set. Superscript indicates the optimal scale's mean number of regions for the respective evaluation measure, approximated to the nearest integer.

both segmentation regimes, including successive examples and failure cases for each method in the superpixel regime, and examples for all evaluated methods in the large segment regime.

\section{Conclusion}

In this work, we presented a new categorization for hierarchical image segmentation into dense and sparse methods, reviewing existing methods that fit into each category and highlighting the benefits and drawbacks of each category in the context of superpixel segmentation.

Noting that the sparse category is underexplored in the literature, we also presented a general scheme for creating new sparse hierarchical methods from existing non-hierarchical superpixel segmentation algorithms using the practical example of our newly proposed Recursive Iterative Spanning Forest (RISF) framework, an extension of the Iterative Spanning Forest (ISF) [15]. We highlight that the scheme is particularly appropriated for the adaptation of existing graph-based and clustering-based superpixel methods. As compared to ISF, RISF has the benefits of generating a multi-scale representation and being faster, with no cost in terms of segmentation quality.

We also showed that superpixel segmentation methods can achieve superior boundary adherence by exploring alternative mid-level segmentation strategies. For that, we presented a new greedy region merging algorithm which, despite being very simple and fast, outperforms the state-of-the-art superpixel segmentation methods when paired with a mid-level segmentation from RISF to generate coarser partitions. The new merging algorithm falls into the dense hierarchical segmentation 


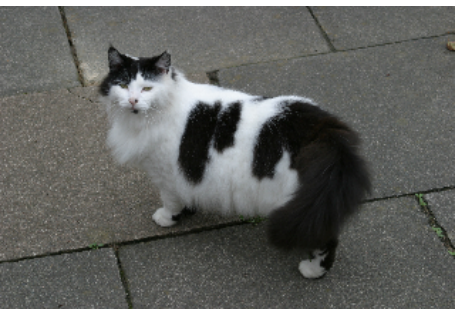

(a) Original

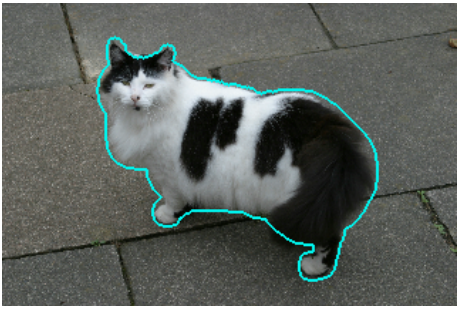

(d) Ground-truth

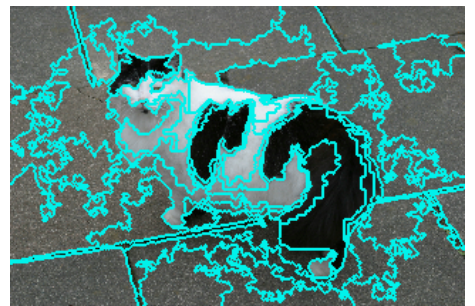

(b) RISF-merge $(N=50)$

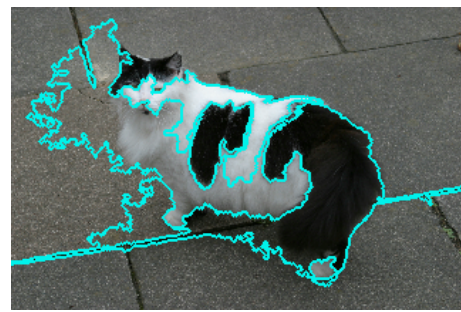

(e) RISF-merge $(N=6)$

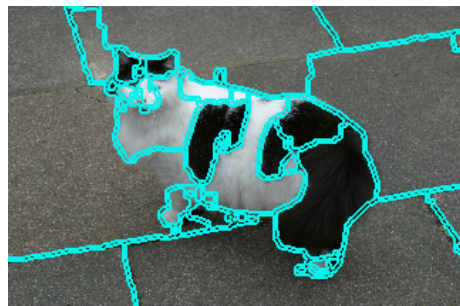

(c) MCG-fast $(N=50)$

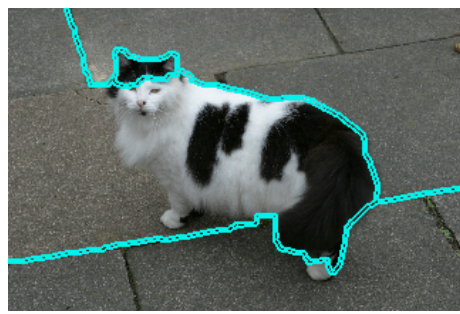

(f) MCG-fast $(N=4)$

Figure 12: Large segment regime comparison of RISF-merge and MCG-fast on a sample of the MSRC dataset. The selected observation scales identified by the number of regions $N$ correspond to the starting point of the large segment regime (b-c) and the OIS number of regions for the BF measure (e-f).

category, being another contribution on that end.

Future work in the sparse category includes studying the performance of the presented sparse hierarchical scheme with other non-hierarchical superpixel methods, and the evaluation of alternative region descriptors for sparse hierarchical segmentation.

In the more general context of superpixel segmentation - including non-hierarchical methods more work should be done on finding new mid-level segmentation strategies that achieve partitions with superior boundary adherence. Regarding our proposed mid-level region merge algorithm in particular, given its exceptional performance when paired with RISF, a better evaluation of its performance when paired with other superpixel segmentation methods is also warranted.

\section{Acknowledgments}

This work was supported by ImmunoCamp, CAPES, CNPq 303808/2018-7, CNPq 310075 / 2019-0, FAPEMIG 00006-18 and FAPESP2014/12236-1. 


\section{References}

${ }_{590}^{2}$ [1] B. Mičušík, J. Košecká, Multi-view superpixel stereo in urban environments, International journal of computer vision 89 (1) (2010) 106-119. doi:10.1007/s11263-010-0327-9.

[2] M. Soltaninejad, G. Yang, T. Lambrou, N. Allinson, T. L. Jones, T. R. Barrick, F. A. Howe, $\mathrm{X}$. Ye, Automated brain tumour detection and segmentation using superpixel-based extremely randomized trees in flair mri, International journal of computer assisted radiology and surgery 12 (2) (2017) 183-203. doi:10.1007/s11548-016-1483-3.

[3] F. Yang, H. Lu, M.-H. Yang, Robust superpixel tracking, IEEE Transactions on Image Processing 23 (4) (2014) 1639-1651. doi:10.1109/TIP.2014.2300823.

[4] Z. Ren, G. Shakhnarovich, Image segmentation by cascaded region agglomeration, in: Proceedings of the IEEE Conference on Computer Vision and Pattern Recognition, 2013, pp. 2011-2018. doi:10.1109/CVPR.2013.262.

[5] M. Maire, S. X. Yu, P. Perona, Hierarchical scene annotation, in: Proceedings of the British Machine Vision Conference, BMVA Press, 2013, p. 84. doi:10.5244/c.27.84.

[6] Z. Liu, W. Zou, L. Li, L. Shen, O. Le Meur, Co-saliency detection based on hierarchical segmentation, IEEE Signal Processing Letters 21 (1) (2013) 88-92.

[7] E. Kim, H. Li, X. Huang, A hierarchical image clustering cosegmentation framework, in: 2012 IEEE Conference on Computer Vision and Pattern Recognition, IEEE, 2012, pp. 686-693. doi:10.1109/CVPR.2012.6247737.

[8] F. Rodrigues, P. Leal, Y. Kenmochi, J. Cousty, L. Najman, S. J. F. Guimarães, Z. K. G. do Patrocínio Jr., Graph-based hierarchical video cosegmentation, in: Image Analysis and Processing - ICIAP 2017 - 19th International Conference, Catania, Italy, September 11-15, 2017, Proceedings, Part I, 2017, pp. 15-26. doi:10.1007/978-3-319-68560-1\_2.

URL https://doi.org/10.1007/978-3-319-68560-1_2

[9] P. Arbelaez, M. Maire, C. Fowlkes, J. Malik, Contour detection and hierarchical image segmentation, IEEE Transactions on Pattern Analysis and Machine Intelligence 33 (5) (2011) 898-916. doi:10.1109/TPAMI.2010.161. 
[10] J. Shi, J. Malik, Normalized cuts and image segmentation, IEEE Transactions on Pattern Analysis and Machine Intelligence 22 (8). doi:10.1109/34.868688.

[11] M.-Y. Liu, O. Tuzel, S. Ramalingam, R. Chellappa, Entropy rate superpixel segmentation, in: Computer Vision and Pattern Recognition (CVPR), Conference on, IEEE, 2011, pp. 20972104. doi:10.1109/CVPR.2011.5995323

[12] X. Wei, Q. Yang, Y. Gong, N. Ahuja, M.-H. Yang, Superpixel hierarchy, IEEE Transactions on Image Processing 27 (10) (2018) 4838-4849. doi:10.1109/TIP.2018.2836300

[13] R. Achanta, A. Shaji, K. Smith, A. Lucchi, P. Fua, S. Süsstrunk, Slic superpixels compared to state-of-the-art superpixel methods, IEEE Transactions on Pattern Analysis and Machine Intelligence 34 (11) (2012) 2274-2282. doi:10.1109/TPAMI.2012.120.

[14] J. Wang, X. Wang, VCells: Simple and efficient superpixels using edge-weighted centroidal voronoi tessellations, IEEE Pattern Analysis and Machine Intelligence 34 (6) (2012) 12411247. doi:10.1109/TPAMI.2012.47.

[15] J. E. Vargas-Muñoz, A. S. Chowdhury, E. B. Alexandre, F. L. Galvão, P. A. V. Miranda, A. X. Falcão, An iterative spanning forest framework for superpixel segmentation, IEEE Transactions on Image Processingdoi:10.1109/TIP.2019.2897941.

[16] K. Nakamura, B.-W. Hong, Hierarchical image segmentation via recursive superpixel with adaptive regularity, Journal of Electronic Imaging 26 (6) (2017) 061602. doi:10.1117/1. JEI. 26.6 .061602 .

[17] Y. Zhou, L. Ju, S. Wang, Multiscale superpixels and supervoxels based on hierarchical edgeweighted centroidal voronoi tessellation, IEEE Transactions on Image Processing 24 (11) (2015) 3834-3845. doi:10.1109/TIP.2015.2449552

[18] F. L. Galvão, A. X. Falcão, A. S. Chowdhury, RISF: Recursive iterative spanning forest for superpixel segmentation, in: Graphics, Patterns and Images (SIBGRAPI), 2018 31st SIBGRAPI Conference on, IEEE, 2018, pp. 408-415. doi:10.1109/SIBGRAPI.2018.00059.

[19] R. Marfil, L. Molina-Tanco, A. Bandera, J. A. Rodríguez, F. Sandoval, Pyramid segmentation algorithms revisited, Pattern Recognition 39 (8) (2006) 1430-1451. doi:10.1016/j.patcog. 2006.02 .017 . 
[20] S. Yin, Y. Qian, M. Gong, Unsupervised hierarchical image segmentation through fuzzy entropy maximization, Pattern Recognition 68 (2017) 245-259. doi:10.1016/j.patcog.2017.03.012.

[21] T. Liu, M. Seyedhosseini, T. Tasdizen, Image segmentation using hierarchical merge tree, 口 IEEE Transactions on Image Processing 25 (10) (2016) 4596-4607. doi:10.1109/TIP.2016. 2592704

[22] D. Stutz, A. Hermans, B. Leibe, Superpixels: An evaluation of the state-of-the-art, Computer Vision and Image Understanding 166 (2018) 1-27. doi:10.1016/j.cviu.2017.03.007.

[23] X. Ren, J. Malik, Learning a classification model for segmentation, in: Proc. IEEE International Conference on Computer Vision (ICCV), IEEE, 2003, p. 10. doi:10.1109/ICCV.2003. 1238308

[24] J. Pont-Tuset, P. Arbelaez, J. T. Barron, F. Marques, J. Malik, Multiscale combinatorial grouping for image segmentation and object proposal generation, IEEE Transactions on Pattern Analysis and Machine Intelligence 39 (1) (2016) 128-140. doi:10.1109/CVPR.2014.49.

[25] S. Beucher, Use of watersheds in contour detection, in: Proceedings of the International Workshop on Image Processing, CCETT, 1979.

[26] A. X. Falcão, J. Stolfi, R. A. Lotufo, The image foresting transform: Theory, algorithms, and applications, IEEE Transactions on Pattern Analysis and Machine Intelligence 26 (1) (2004) 19-29. doi:10.1109/TPAMI.2004.1261076.

[27] K. C. Ciesielski, A. X. Falcão, P. A. V. Miranda, Path-value functions for which dijkstra's algorithm returns optimal mapping, Journal of Mathematical Imaging and Visiondoi:10. 1007/s10851-018-0793-1

[28] P. F. Felzenszwalb, D. P. Huttenlocher, Efficient graph-based image segmentation, International Journal of Computer Vision 59 (2) (2004) 167-181. doi:10.1023/B:VISI.0000022288. 19776.77 .

[29] W. G. Kropatsch, Y. Haxhimusa, Grouping and segmentation in a hierarchy of graphs, in: Computational Imaging II, Vol. 5299, International Society for Optics and Photonics, 2004, pp. 193-204. doi:10.1117/12.543421. 
[30] M. Grundmann, V. Kwatra, M. Han, I. Essa, Efficient hierarchical graph-based video segmentation, in: Computer Vision and Pattern Recognition (CVPR), Conference on, IEEE, 2010, pp. 2141-2148. doi:10.1109/CVPR.2010.5539893.

[31] K. J. F. Souza, A. A. Araújo, Z. K. G. Patrocínio Jr, S. J. F. Guimarães, Graph-based hi-

[38] L. Guigues, J. P. Cocquerez, H. Le Men, Scale-sets image analysis, International Journal of Computer Vision 68 (3) (2006) 289-317. doi:10.1007/s11263-005-6299-0. 
[39] R. E. Tarjan, Efficiency of a good but not linear set union algorithm, Journal of the ACM (JACM) 22 (2) (1975) 215-225. doi:10.1145/321879.321884.

[40] J. B. Kruskal, On the shortest spanning subtree of a graph and the traveling salesman problem, Proceedings of the American Mathematical society 7 (1) (1956) 48-50. doi: 10.1090/S0002-9939-1956-0078686-7.

[41] J. Shotton, J. Winn, C. Rother, A. Criminisi, Textonboost: Joint appearance, shape and context modeling for multi-class object recognition and segmentation, in: European Conference on Computer Vision, Springer, 2006, pp. 1-15. doi:10.1007/11744023_1.

[42] L. A. Mansilla, P. A. V. Miranda, F. A. Cappabianco, Image segmentation by image foresting transform with non-smooth connectivity functions, in: Graphics, Patterns and Images (SIBGRAPI), 2013 26th SIBGRAPI-Conference on, IEEE, 2013, pp. 147-154. doi: 10.1109/SIBGRAPI.2013.29

[43] T. Malisiewicz, A. A. Efros, Improving spatial support for objects via multiple segmentations, in: Proceedings of the British Machine Vision Conference, BMVA Press, 2007, pp. 55.1-55.10. doi:10.5244/C.21.55.

[44] P. Buyssens, M. Toutain, A. Elmoataz, O. Lézoray, Eikonal-based vertices growing and iterative seeding for efficient graph-based segmentation, in: 2014 IEEE International Conference on Image Processing (ICIP), IEEE, 2014, pp. 4368-4372. doi:10.1109/ICIP.2014.7025886

[45] P. Neubert, P. Protzel, Superpixel benchmark and comparison, in: Proc. Forum Bildverarbeitung, 2012, pp. 1-12.

[46] M. Meilă, Comparing clusterings - an information based distance, Journal of multivariate analysis 98 (5) (2007) 873-895. doi:10.1016/j.jmva.2006.11.013

[47] R. Unnikrishnan, C. Pantofaru, M. Hebert, A measure for objective evaluation of image segmentation algorithms, in: Computer Vision and Pattern Recognition Workshops (CVPRW), IEEE Computer Society Conference on, IEEE, 2005, pp. 34-34. doi:10.1109/CVPR.2005.390 